\title{
Fully coherent follow-up of continuous gravitational-wave candidates
}

\author{
M. Shaltev, R. Prix \\ Albert-Einstein-Institut, Callinstr. 38, 30167 Hannover, Germany \\ (Dated: Mon Mar 11 10:10:31 $2013+0100$ ) \\ (LIGO-P1200185-v2) \\ (MmitID: d849404-CLEAN)
}

\begin{abstract}
The search for continuous gravitational waves from unknown isolated sources is computationally limited due to the enormous parameter space that needs to be covered and the weakness of the expected signals. Therefore semi-coherent search strategies have been developed and applied in distributed computing environments such as Einstein@Home, in order to narrow down the parameter space and identify interesting candidates. However, in order to optimally confirm or dismiss a candidate as a possible gravitational-wave signal, a fully-coherent follow-up using all the available data is required.

We present a general method and implementation of a direct (2-stage) transition to a fullycoherent follow-up on semi-coherent candidates. This method is based on a grid-less Mesh Adaptive Direct Search (MADS) algorithm using the $\mathcal{F}$-statistic. We demonstrate the detection power and computing cost of this follow-up procedure using extensive Monte-Carlo simulations on (simulated) semi-coherent candidates from a directed as well as from an all-sky search setup.
\end{abstract}

\section{INTRODUCTION}

Continuous gravitational waves (CWs) are expected to be emitted from rapidly spinning non-axisymmetric compact objects, e.g. neutron stars. The computational cost of a coherent matched-filtering detection statistic, such as the $\mathcal{F}$-statistic [1, is small provided the parameters of the source (i.e. sky position $\alpha, \delta$, frequency $f$, frequency derivatives $\dot{f}, \ldots$ ) are known. However, wide parameter-space searches for unknown sources quickly become computationally prohibitive, due to the large number of points in parameter space (templates) that need to be searched [2].

In order to first reduce the parameter space to smaller, more promising regions, semi-coherent search techniques have been developed [3 6] and are currently being used [7. 8], for example in the Einstein@Home distributed computing environment 9. In a semi-coherent search the total amount of data $T$ is divided into $N$ shorter segments of duration $\Delta T$. The coherent statistics from the individual segments are combined to a new semi-coherent statistic. At fixed computing cost these semi-coherent methods are (typically) more sensitive than fully-coherent searches [10].

Structuring a wide parameter-space search into hierarchical stages, which increasingly concentrate computational power onto the more promising regions of parameter space, was first described in [2] and elaborated further in [3], where a two-stage semi-coherent hierarchical search was considered. An extended hierarchical scheme with an arbitrary number of semi-coherent stages and a final fully-coherent stage was studied numerically in 4, which concluded that three semi-coherent stages will typically be a good choice. In [11] and [12] the use of an optimization procedure has been considered in the process of estimation of the source parameters, once a candidate is considered as a detection. In both cases, however, no practical method or implementation was provided for the systematic coherent follow-up of semi-coherent candidates.

The aim of the present work is to introduce such a coherent follow-up search strategy and implementation. This is achieved by exploring the parameter space around a semi-coherent candidate using a Mesh Adaptive Direct Search (MADS) algorithm. Using this method, we find that a fully-coherent follow-up (using all of the available data) of initial semi-coherent candidates is computationally feasible.

This paper is organized as follows: in Section II we describe the relevant basic concepts in CW searches, in Section III we propose a search strategy for the systematic follow-up of CW candidates, in Section IV we present a Monte-Carlo study and in Section $\mathrm{V}$ we discuss the results.

\section{Notation}

We distinguish a quantity $Q$ when referring to a fullycoherent stage using a tilde, $\widetilde{Q}$ and when referring to a semi-coherent stage using an overhat, $\widehat{Q}$. Averaging over segments is denoted by an overbar, $\bar{Q}$.

\section{CONTINUOUS GRAVITATIONAL WAVES}

Continuous gravitational-wave signals are quasimonochromatic and sinusoidal in the source frame and undergo phase- and amplitude-modulation due to the diurnal and orbital motion of the detectors. The phase evolution of the signal at a detector can be approximated 
as 1 .

$$
\begin{aligned}
\Phi(t) & \approx \Phi_{0}+2 \pi \sum_{k=0}^{s} \frac{f^{(k)}\left(t_{0}\right)\left(t-t_{0}\right)^{k+1}}{(k+1) !} \\
& +2 \pi \frac{\mathbf{r}(t)}{c} \mathbf{n} \sum_{k=0}^{s} \frac{f^{(k)}\left(t_{0}\right)\left(t-t_{0}\right)^{k}}{k !}
\end{aligned}
$$

where $\Phi_{0}$ is the initial phase, $f^{(k)} \equiv \frac{d^{k} f}{d t^{k}}$ are the derivatives of the signal frequency $f$ at the solar system barycenter (SSB) at reference time $t_{0}, c$ is the speed of light, $\mathbf{r}(t)$ is the vector pointing from the SSB to the detector and $\mathbf{n}$ is the unit vector pointing from the SSB to the gravitational-wave source.

\section{A. Detection statistic}

Following [1, 13] the gravitational-wave response of a detector can be expressed as a sum over four (detectorindependent) amplitude parameters multiplying four (detector-dependent) basis waveforms. The amplitude parameters can be analytically maximized over and the resulting detection statistic, known as the $\mathcal{F}$-statistic, is therefore a function only of the template "phase parameters" $\lambda \equiv\{\alpha, \delta, f, \dot{f}, \ldots\}$, where $\alpha$ (right ascension) and $\delta$ (declination) denote the sky position of the source.

In the presence of a signal the fully-coherent detection statistic $2 \mathcal{F}$ follows a non-central $\chi^{2}$-distribution with 4 degrees of freedom and a non-centrality parameter given by the squared signal to noise ratio (SNR), $\rho^{2}$. The expectation value is therefore

$$
E[2 \mathcal{F}]=4+\rho^{2},
$$

with variance

$$
\sigma^{2}[2 \mathcal{F}]=2\left(4+2 \rho^{2}\right) .
$$

On the other hand, in the semi-coherent approach we divide the available data into $N$ segments of duration $\Delta T$ and combine the individual coherent statistics of the segments to compute a semi-coherent statistic, namely

$$
\overline{2 \mathcal{F}}(\lambda)=\frac{1}{N} \sum_{k=1}^{N} 2 \mathcal{F}_{k}(\lambda),
$$

where $2 \mathcal{F}_{k}$ is the coherent $\mathcal{F}$-statistic in segment $k$. The quantity $N \overline{2 \mathcal{F}}$ follows a non-central $\chi^{2}$-distribution with $4 N$ degrees of freedom, thus the expectation value of $\overline{2 \mathcal{F}}$ is

$$
E[\overline{2 \mathcal{F}}]=4+\overline{\rho^{2}}
$$

with variance

$$
\sigma^{2}[\overline{2 \mathcal{F}}]=\frac{2}{N}\left(4+2 \overline{\rho^{2}}\right),
$$

where $\overline{\rho^{2}}$ is the average $\mathrm{SNR}^{2}$ over all segments, i.e.

$$
\overline{\rho^{2}}=\frac{1}{N} \sum_{k=1}^{N} \rho_{k}^{2},
$$

and $\rho_{k}^{2}$ denotes the $\mathrm{SNR}^{2}$ in segment $k$.

\section{B. Mismatch and Fisher matrix}

A search for sources with unknown signal parameters implies a loss of detection power compared to the perfectly-matched case. To quantify this we use the notion of mismatch $\mu$, as first introduced in [14, 15. This is defined as the fractional loss of expected $\mathrm{SNR}^{2}$ at some parameter space point $\lambda$ compared to the expectation $\rho^{2}\left(\lambda_{s}\right)$ at the signal location $\lambda_{s}$, namely

$$
\mu \equiv \frac{\rho^{2}\left(\lambda_{s}\right)-\rho^{2}(\lambda)}{\rho^{2}\left(\lambda_{s}\right)},
$$

such that $\mu \in[0,1]$. Taylor expansion in small offsets $\Delta \lambda=\lambda-\lambda_{s}$ around the signal location yields

$$
\mu \equiv g_{i j}\left(\lambda_{s}\right) \Delta \lambda^{i} \Delta \lambda^{j}+\mathcal{O}\left(\Delta \lambda^{3}\right),
$$

where implicit summation over repeated parameter-space indices $i, j$ applies, and the symmetric positive-definite matrix $g_{i j}$ is commonly referred to as the parameterspace metric.

Neglecting higher order terms, one often uses the "metric mismatch approximation", namely

$$
\mu^{*} \equiv g_{i j}\left(\lambda_{s}\right) \Delta \lambda^{i} \Delta \lambda^{j},
$$

as a distance measure, with a range $\mu^{*} \in[0, \infty)$. This metric mismatch $\mu^{*}$ plays an important role in grid-based searches, where one typically constructs template banks in such way that the mismatch of any putative signal and the "closest" template is bounded by a maximal mismatch $m$, i.e.

$$
\mu^{*} \leq m
$$

everywhere in the template bank.

In the presence of noise, $\mu$ as defined in Eq. 8 is not directly accessible, and we therefore introduce a related quantity, namely the fractional loss of measured $\mathrm{SNR}^{2}$, namely

$$
\check{\mu} \equiv \frac{2 \mathcal{F}\left(\lambda_{s}\right)-2 \mathcal{F}(\lambda)}{2 \mathcal{F}\left(\lambda_{s}\right)-4} .
$$

Note that $\check{\mu} \leq 1$, but contrary to $(8)$ it can also be (slightly) negative, as we can have $2 \mathcal{F}\left(\lambda_{s}\right)<2 \mathcal{F}(\lambda)$ due to noise.

For semi-coherent searches the metric is found [3] as the average of the fully-coherent metrics over all the segments, namely

$$
\widehat{g}_{i j}(\lambda)=\frac{1}{N} \sum_{k=1}^{N} g_{i j, k}(\lambda),
$$


where $\widetilde{g}_{i j, k}$ is the coherent metric $(9)$ in segment $k$.

A standard tool for parameter estimation is provided by the Fisher information matrix, which characterizes the statistical uncertainty of the maximum-likelihood estimators (MLE) $\lambda_{\mathrm{MLE}}^{i}$ for the signal parameters $\lambda_{s}^{i}$. This can be formulated [16 18] as the well-known Cramer-Ráo lower bound on the variance of an unbiased MLE (i.e. $\left.E\left[\lambda_{\mathrm{MLE}}^{i}\right]=\lambda_{s}^{i}\right)$, namely

$$
\sigma^{2}\left[\lambda_{\mathrm{MLE}}^{i}\right] \geq\left\{\Gamma^{-1}\right\}^{i i}
$$

where the matrix $\left\{\Gamma^{-1}\right\}^{i j}$ denotes the inverse of the Fisher matrix $\Gamma_{i j}$, which is closely related (e.g. [17]) to the metric $g_{i j}$, namely

$$
\Gamma_{i j}=\rho^{2} g_{i j}
$$

A semi-coherent search over $N$ segments can be considered as $N$ different measurements, thus the semi-coherent Fisher matrix yields 19

$$
\widehat{\Gamma}=\sum_{k=1}^{N} \Gamma_{i j, k}
$$

Assuming constant $\mathrm{SNR}^{2}$ for the different segments we can rewrite (16) in terms of the semi-coherent metric (13), namely

$$
\widehat{\Gamma}=N \overline{\rho^{2}} \widehat{g}_{i j}
$$

and thus

$$
\left\{\widehat{\Gamma}^{-1}\right\}^{i j}=\frac{\widehat{g}^{i j}}{N \overline{\rho^{2}}}
$$

where $\widehat{g}^{i j}$ is the inverse matrix of $\widehat{g}_{i j}$.

\section{Computing cost}

The computing cost $C$ of a fully-coherent (or an ideal semi-coherent [10]) search is primarily due to the computation of the $\mathcal{F}$-statistic over all the templates. For a search over $\mathcal{N}$ templates using $N$ segments of data from $N_{\text {det }}$ detectors [10], the computing cost $C$ is

$$
C=N \mathcal{N} N_{\text {det }} c_{1}
$$

where $c_{1}$ is the implementation-dependent computing cost for a single template, segment and detector. A method of $\mathcal{F}$-statistic computation based on short Fourier transforms (SFTs) 20] of length $T_{\mathrm{SFT}}$ is currently widely used in CW searches and will be considered in the present work. The cost per template in this case is proportional to the segment duration, namely

$$
c_{1}^{\mathrm{SFT}}=c_{0}^{\mathrm{SFT}} \frac{\Delta T}{T_{\mathrm{SFT}}},
$$

where $c_{0}^{\mathrm{SFT}}$ is implementation- and hardware-dependent fundamental computing cost per SFT. Using the total number of SFTs

$$
N_{\mathrm{SFT}}=N N_{\mathrm{det}} \frac{\Delta T}{T_{\mathrm{SFT}}},
$$

we can write the total computing cost 19 of the SFTmethod as

$$
C=\mathcal{N} N_{\mathrm{SFT}} c_{0}^{\mathrm{SFT}}
$$

In grid-based searches the number of templates required to cover the search parameter space $\mathbb{P}$ is given by the general expression [21, 22,

$$
\mathcal{N} \equiv \theta_{n} m^{-n / 2} \int_{\mathbb{P}} d^{n} \lambda \sqrt{\operatorname{det} g},
$$

where $\theta$ is the normalized lattice thickness, $n$ is the number of search dimensions, $m$ is the maximal templatebank mismatch (11) and det $g$ is the determinant of the parameter space metric (9). The normalized thickness is a constant depending on the grid structure, e.g. for a hyper-cubic lattice $\theta_{\mathbb{Z}_{n}}=n^{n / 2} 2^{-n}$. The metric $g_{i j}$ depends strongly on the duration $\Delta T$ and the number of segments $N$, in such a way that longer observation times typically require a (vastly) increased number of templates 2].

\section{COHERENT FOLLOW-UP OF SEMI-COHERENT CANDIDATES}

\section{A. Basic two-stage search strategy}

Here we introduce a simple two-stage strategy for following-up candidates from semi-coherent searches. In the first stage, called refinement, we employ a finer search using the semi-coherent statistic $\overline{2 \mathcal{F}}$ to improve the initial maximum-likelihood estimator. In the second stage, called zoom, we apply the fully-coherent statistic $\widetilde{2 \mathcal{F}}$ using all the data $T$, in order to test whether the candidate is inconsistent with Gaussian noise and if it further agrees with the signal model.

The motivation for this 2-stage approach can be seen from an example 2-D search grid shown in Fig. 11. The search templates are generally placed such that a putative signal $\lambda_{s}$ will be recovered with a loss of SNR bounded by a maximal mismatch $m$, as given in Eq. (11), namely

$$
g_{i j} \Delta \lambda^{i} \Delta \lambda^{j} \leq m
$$

where equality defines an ( $n$-dimensional) iso-mismatch ellipse. The initial semi-coherent search will yield "candidates" $\widehat{\lambda}_{c}$ for which the statistic $2 \overline{\mathcal{F}}$ exceeds a certain threshold and is higher than neighboring templates.

The initial refinement stage of our follow-up strategy therefore consists in finding the (nearby) parameterspace point $\widehat{\lambda}_{\text {MLE }}$ of the actual (local) maximum in the 


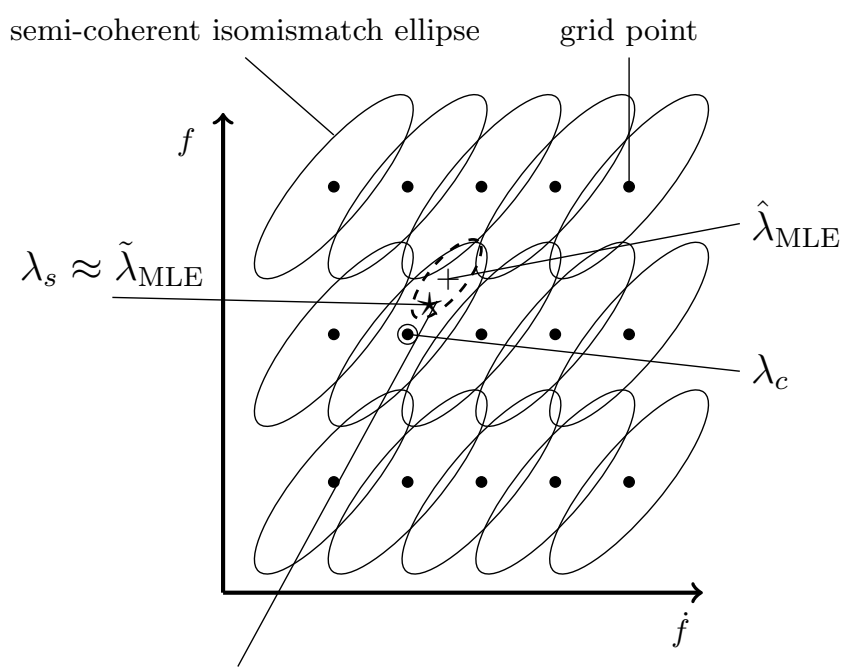

semi-coherent Fisher ellipse

FIG. 1: 2-D search grid in $\{f, \dot{f}\}$ space. The black dots are the search templates, placed such that the loss of SNR on any putative signal $\lambda_{s}$ will be bounded by a maximal mismatch $m$, which defines the semi-coherent iso-mismatch ellipses. The semi-coherent Fisher ellipse centered on the MLE $\widehat{\lambda}_{\mathrm{MLE}}$ is used to constrain the zoom parameter space. The aim of the zoom stage is to find $\widetilde{\lambda}_{\mathrm{MLE}}$

statistic $2 \overline{\mathcal{F}}(\widehat{\lambda})$ (which is smooth function of $\widehat{\lambda}$ ), referred to as the maximum-likelihood estimator (MLE). This can be achieved simply by a denser placement of templates using the original statistic, i.e. by keeping the search setup unchanged in terms of the number and length of segments.

In the zoom stage we fully-coherently search the Fisher ellipse centered on the semi-coherent MLE $\widehat{\lambda}_{\mathrm{MLE}}$. This defines the parameter-space region that should contain the signal location $\lambda_{s}$ with confidence corresponding to $n_{\mathrm{B}}$ standard deviations, i.e.

$$
\widehat{\Gamma}_{i j} \delta \lambda^{i} \delta \lambda^{j} \leq n_{\mathrm{B}}^{2}
$$

where $\delta \lambda^{i}=\widehat{\lambda}_{\mathrm{MLE}}^{i}-\lambda_{s}^{i}$. Note that the Fisher ellipse actually describes the fluctuations of the maximum-likelihood estimator $\widehat{\lambda}_{\mathrm{MLE}}$ for given signal location. However, provided the likelihood-manifold is not strongly curved, this also describes our uncertainty of the signal location for given MLE $\widehat{\lambda}_{\text {MLE }}$, as indicated in Fig. 2. The zoom stage will yield the fully-coherent maximum-likelihood estimator $\widetilde{\lambda}_{\mathrm{MLE}}$, which represents our best estimate for the signal parameters $\lambda_{s}$. Thus the two-stage search strategy corresponds to the transition

$$
\widehat{\lambda}_{c} \stackrel{\text { refinement }}{\longrightarrow} \widehat{\lambda}_{\mathrm{MLE}} \stackrel{\text { zoom }}{\longrightarrow} \widetilde{\lambda}_{\mathrm{MLE}} \approx \lambda_{s} .
$$

In the following we use a subscript $R$ to denote quantities

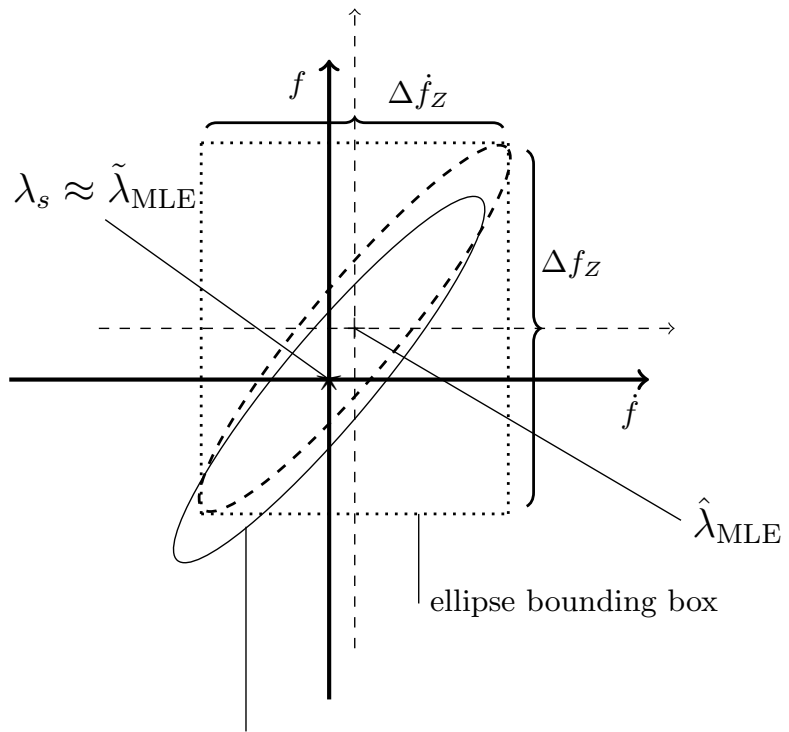

semi-coherent Fisher ellipse

FIG. 2: 2-D example: Fisher ellipse 25) defining the zoom search space, centered on the semi-coherent MLE $\widehat{\lambda}_{\text {MLE }}$. The extents $\{\Delta f, \Delta \dot{f}\}$ of the bounding box are given by Eq. (27).

in the refinement stage and a subscript $Z$ for quantities in the zoom stage.

The search volume for the refinement stage depends on the template bank construction of the original semicoherent search. Ideally one iso-mismatch ellipse corresponding to the original template-bank construction (see Fig. (1) should be sufficient. In practice, however, it might often be neccessary to use several grid spacings in each direction, if the template bank was not originally constructed in a strictly metric way. In this case the exact number of grid spacings will have to be empirically determined in a Monte Carlo study.

\section{Bounding box and volume of $n$-dimensional ellipses}

In the following it will be useful to express the bounding box and volume of an $n$-dimensional ellipse, namely for the iso-mismatch ellipse of Eq. 24) and the Fisher ellipse of Eq. 25]. The general form of the $n$-dimensional ellipse equation is

$$
G_{i j} d \lambda^{i} d \lambda^{j}=R^{2}
$$

where $G_{i j}$ is a positive-definite symmetric matrix. The extents $\Delta \lambda^{i}$ of a bounding box along coordinate axes $\lambda^{i}$ (as indicated in Fig. 2) can be obtained from the diagonal elements of the inverse matrix, $\left\{G^{-1}\right\}^{i j}$, namely

$$
\Delta \lambda^{i}=2 R \sqrt{\left\{G^{-1}\right\}^{i i}} .
$$


The ellipse coordinate volume is expressible via the matrix determinant, $\operatorname{det} G$, namely

$$
V=\frac{R^{n}}{\sqrt{\operatorname{det} G}} \mathcal{V}_{n},
$$

where $\mathcal{V}_{n}=\frac{\pi^{n / 2}}{\Gamma(1+n / 2)}$ is the volume of unit $n$-ball.

\section{B. Classification of zoom outcomes}

Assuming a real CW signal, we can estimate the range of expected values of the fully-coherent zoom $\mathcal{F}$-statistic in $\tilde{\lambda}_{s}$. From Eq. (5) we can obtain a (rough) estimate of the average-SNR ${ }^{2}$ from the measured average$\mathrm{SNR}^{2}$ of the semi-coherent maximum-likelihood estimator, namely

$$
\bar{\rho}_{\mathrm{MLE}} \approx \overline{2 \mathcal{F}}_{\mathrm{MLE}}-4
$$

The $\mathrm{SNR}^{2}$ of the fully-coherent search is linear in the number of segments $N$, i.e.

$$
\widetilde{\rho}^{2}=N \overline{\rho^{2}}{ }_{\mathrm{MLE}}
$$

Substitution of the above expression in Eq. 22 yields the expectation for the fully-coherent matched filter in $\widetilde{\lambda}_{\mathrm{MLE}}$, namely

$$
\widetilde{2 \mathcal{F}}_{o} \equiv E[\widetilde{2 \mathcal{F}}] \approx 4+N{\overline{\rho^{2}}}_{\mathrm{MLE}}
$$

Further substitution of Eq. 30 in Eq. (3) yields the corresponding variance as

$$
\sigma_{o}^{2} \equiv \sigma^{2}[\widetilde{2 \mathcal{F}}] \approx 2\left(4+2 N \overline{\rho^{2}}{ }_{\text {MLE }}\right)
$$

These quantities are useful for defining what we mean by confirming a CW signal.

Note that the uncertainty in the original SNRestimation in Eq. 29 results in a distribution around the final estimate of Eq. 31) that is wider than estimated by Eq. (32). This effect can be computed analytically and empirically, and is found to amount to about a factor of 2.

Depending on the maximal $\widetilde{2 \mathcal{F}}$ value found in the final zoom stage, we can distinguish 3 possible outcomes:

- Consistency with Gaussian noise $(G)$ - the fullycoherent $\widetilde{2 \mathcal{F}}$ value does not exceed a threshold

$$
\widetilde{2 \mathcal{F}}<\widetilde{2 \mathcal{F}}_{\text {th }}^{(G)}
$$

where $\widetilde{2 \mathcal{F}}_{\text {th }}^{(G)}$ is chosen to corresponds to some (small) false-alarm probability $p_{\mathrm{fA}}$ in Gaussian noise.

For example, a threshold $\widetilde{2 \mathcal{F}}_{\text {th }}^{(G)}=60$ corresponds to a very small false-alarm probability of order $10^{-12}$ in a single template, as given by Eq. 43.)
- Non-Gaussian origin $(\neg G)$ - the candidate is loud enough to be inconsistent with Gaussian noise at the false-alarm probability $p_{\mathrm{fA}}$, i.e.

$$
\widetilde{2 \mathcal{F}} \geq \widetilde{2 \mathcal{F}}_{\text {th }}^{(G)}
$$

- We define signal recovery $(S)$ as a subclass of $\neg G$, namely if the final zoomed candidate $\widetilde{2 \mathcal{F}}$ exceeds the Gaussian-noise threshold $\widetilde{2 \mathcal{F}}_{\text {th }}^{(G)}$ and falls into the predicted signal interval given by Eqs. (31) and (32) (at some confidence level). We can write this as

$$
\widetilde{2 \mathcal{F}}_{\text {th }}^{(S)}<\widetilde{2 \mathcal{F}}<\widetilde{2 \mathcal{F}}_{\max }^{(S)}
$$

where $\widetilde{2 \mathcal{F}}_{\text {th }}^{(S)} \equiv \max \left\{\widetilde{2 \mathcal{F}}_{\text {th }}^{(G)}, \widetilde{2 \mathcal{F}}_{o}-n_{u} \sigma_{o}\right\}$, and $\widetilde{2 \mathcal{F}}_{\text {max }}^{(S)} \equiv \widetilde{2 \mathcal{F}}_{o}+n_{u} \sigma_{o}$, where $n_{u}$ determines the desired confidence level. In this work we consider $n_{u}=6$, which corresponds roughly to a confidence of $\sim 99.6 \%$.

Note that there can be cases where a zoomed candidate ends up in $\neg G$ but does not make it into the signal recovery $(S)$ band, e.g. typically $\widetilde{2 \mathcal{F}}_{\text {th }}^{(G)}<\widetilde{2 \mathcal{F}}<\widetilde{2 \mathcal{F}}_{\text {th }}^{(S)}$. There can be different reasons for this, e.g. the search algorithm converged to a secondary maximum in the refinement or zoom stage, the signal model deviates from reality and requires modification, or the "signal" found is of non astrophysical origin (eg a detector-noise artifact). Generally further investigation will be required for all candidates falling into the non-Gaussian category $(\neg G)$.

\section{Grid-based computing-cost of the zoom stage}

We do not consider a grid-based follow-up method in this paper, but it is instructive to estimate the corresponding computing-cost for later comparison. To estimate the number of templates required for the fullycoherent search, we can use Eq. 28 to compute the volume of the follow-up Fisher ellipse, Eq. (25), and divide it by the volume covered by one coherent template, Eq. (24). Namely, the Fisher-ellipse volume is given by

$$
\widehat{V}=\frac{n_{\mathrm{B}}^{n}}{\left(N \overline{\rho^{2}}\right)^{n / 2} \sqrt{\operatorname{det} \widehat{g}}} \mathcal{V}_{n},
$$

while the coherent template-volume at mismatch $m$ is

$$
\widetilde{V}=\frac{m^{n / 2}}{\sqrt{\operatorname{det} \widetilde{g}}} \mathcal{V}_{n}
$$

therefore we can estimate then number of template as

$$
\mathcal{N} \approx \frac{\widehat{V}}{\widetilde{V}}=\frac{n_{\mathrm{B}}^{n}}{\left(N \overline{\rho^{2}}\right)^{n / 2} m^{n / 2}} \frac{\sqrt{\operatorname{det} \widetilde{g}}}{\sqrt{\operatorname{det} \widehat{g}}} .
$$


Consider a follow-up of a candidate from a directed $n=2$ search in $\{f, \dot{f}\}$ (e.g. see Fig. 1). Assuming a semicoherent search using $N=200$ segments of $\Delta T=1 \mathrm{~d}$ duration without gaps, and a fully-coherent observation time of $T=200 \mathrm{~d}$. Using the expressions found in [23, the determinants of the two-dimensional coherent and the semi-coherent metrics are found as

$$
\begin{aligned}
\sqrt{\operatorname{det} \widetilde{g}} & =\pi^{2} T^{3} \frac{1}{540}, \\
\sqrt{\operatorname{det} \widehat{g}} & =\pi^{2} \Delta T^{3} \frac{\gamma(N)}{540},
\end{aligned}
$$

where $\gamma \approx \sqrt{5} N$ is the spindown refinement factor. Putting everything together in Eq. (38), we obtain

$$
\mathcal{N} \approx \frac{n_{\mathrm{B}}^{2} N}{\sqrt{5} \overline{\rho^{2}} m}
$$

where we used $N=T / \Delta T$. For a signal with $\overline{\rho^{2}}=$ $1, n_{\mathrm{B}}=24^{1}$ and $m=0.1$ the number of templates is therefore $\mathcal{N} \approx 5.1 \times 10^{5}$. Thus, using Eq. 22 for 2 detectors and the SFT method with $T_{\mathrm{SFT}}=1800 \mathrm{~s}$, the zoom computing cost is $C \approx 11$ min per candidate, where we used the fundamental computing $\operatorname{cost} c_{0}^{\mathrm{SFT}}=$ $7 \times 10^{-8} s[10$.

In the more general case where the sky position of the source is also unknown, the number of sky points typically scales at least quadratically with the observation time [23, 24] (for coherent integration longer than few days), thus generally resulting in completely prohibitive computational requirements for grid-based follow-up searches. In particular, extending the directed search example from the previous paragraph to an all-sky follow-up would require $\mathcal{N}_{\text {sky }} \approx 1.3 \times 10^{6}$ sky points $^{2}$, or a total of $\mathcal{N} \approx 6.8 \times 10^{11}$ templates.

For comparison, using the grid-less search algorithm discussed in the next sections, it is possible to coherently follow up 2-D directed candidates in less than 2 minutes, see Fig. $4 \mathrm{~d}$, and all-sky candidates in about 1 hour per candidate, see Fig. 5d.

\section{Mesh Adaptive Direct Search (MADS)}

A significant difference between the hierarchical search strategies discussed in [2 4] and this work is the method of template bank construction at the different stages. Namely, we consider a grid-less method for exploring the parameter space.

\footnotetext{
1 This large $n_{\mathrm{B}}$ value is found to contain the signal location in more than $98 \%$ of the cases even for weak signals, where the Fisher-matrix may be a poor predictor, see [16].

2 The number of sky templates has been estimated by numerical computation of the sky part of the metrics $\widehat{g}$ and $\widetilde{g}$ using FstatMetric_v2 from LALSUITE 25, see also [17.
}

The MADS class of algorithms for derivative-free optimization has been first introduced in [26] and further developed in 27] and 28, among others. In this subsection we only introduce some of the control parameters of the algorithm required in the construction of MADSbased $\mathcal{F}$-statistic searches, for an in-depth treatment and proofs we refer the reader to the cited publications.

MADS consist of the iteration of two steps, called search and poll, in which trial points are constructed and evaluated in order to find an extremum. In the search step any strategy can be applied to construct trial points. In this work we use quadratic models (quadratic form) to approximate the objective function from a sample of objective values [28. If the local exploration in the search step fails to generate a new solution, a set of poll points is generated using a stochastic or deterministic method. Stochastic means that the poll points are generated randomly [26], where deterministic refers to the usage of pseudo-random Halton sequences [27. However both methods generate points which form a dense set in the unit sphere after an infinite number of iterations. For a given starting point $\lambda_{c}$ with parameter space boundaries $\Delta \lambda_{\mathrm{B}}$, initial step sizes $d \lambda$ and a method for generation of poll points, the discretization of the parameter space $\Delta_{k}^{m}$ at iteration $k$ is governed by a fixed rational number $u_{b}>1$ and the coarsening $w^{+} \geq 0$ and refining $w^{-} \leq-1$ exponents. If the current iteration generates a better solution, the discretization in the next iteration is coarser, namely $\Delta_{k+1}^{m}=u_{b}^{w^{+}} \Delta_{k}^{m}$, otherwise $\Delta_{k+1}^{m}=u_{b}^{w^{-}} \Delta_{k}^{m}$ [26]. The algorithm stops if an improved solution cannot be found or the total number of evaluated parameter space points $p$ reaches some given maximum $p_{\max }$.

\section{E. MADS-based follow-up algorithm}

From the point of view of the MADS algorithm, the function to optimize is a black-box requiring some input to produce a single output value. The black-box in our case is either the computation of the semi-coherent $\mathcal{F}$ statistic $\overline{2 \mathcal{F}}$ of Eq. $4(1)$ in the refinement, or the fullycoherent $\mathcal{F}$-statistic $2 \mathcal{F}$ in the zoom stage. In order to minimize the possibility of convergence to secondary maxima, we run multiple instances of the MADS search in each stage varying the mesh coarsening exponent $w^{+}$. The minimal $w_{\min }^{+}$and maximal $w_{\max }^{+}$coarsening exponent determine the number of MADS steps in each pass, namely $n_{\text {steps }}=w_{\max }^{+}-w_{\min }^{+}+1$. Thus we consider our search algorithm composed of several instances of MADS, see Fig. 3. The input of the search algorithm is the candidate $\lambda_{c}$ to follow-up, the search boundaries $\Delta \lambda_{R / Z}$ around the candidate and a set of MADS input parameters, namely $\left\{d \lambda, u_{b}, w_{\min }^{+}, w_{\max }^{+}, w^{-}\right\}$. In the zoom stage the search boundaries $\left(\Delta \lambda_{Z}\right)$ are estimated from the bounding box of the Fisher ellipse, using Eq. (27). For the refinement stage the search boundaries $\left(\Delta \lambda_{R}\right)$ generally have to be determined depending on the template-bank setup of the original semi-coherent search. 




FIG. 3: MADS-based search algorithm with 4 passes, where $\lambda_{0}=\lambda_{c}$ in the refinement stage and $\lambda_{0}=\widehat{\lambda}_{\mathrm{MLE}}$ in the zoom stage.

Note, however, that the bounding boxes $\Delta \lambda$ only serve as a necessary input parameter to the NOMAD search algorithm, while the effective MADS search region can be further reduced by rejecting points that do not satisfy a given constraint. For example, the effective search region in the zoom stage always consists of the Fisher ellipse Eq. 25).

The initial step sizes $d \lambda^{i}$ are also empirically determined, typically as some fraction of the search boundary $\Delta \lambda_{R / Z}^{i}$.

We propose a 4 pass algorithm with equal (for simplicity) number of steps $n_{\text {steps }}$ in each pass, however with different starting point and method of trial-point generation:

- 1st pass - starting point $\lambda_{c}$, deterministic point generation,
- 2nd pass - starting point $\lambda_{c}$, stochastic point generation,

- 3rd pass - starting point loudest template from the first 2 passes, deterministic point generation,

- 4th pass - starting point from the vicinity of the loudest point from the first 2 passes, stochastic point generation.

In the zoom stage we terminate the search as soon as the loudest point of the current iteration satisfies the signal-confirmation condition $(S)$ of Eq. (35). In lowerdimensional cases, such as the directed search considered later, a single pass is therefore often found to be sufficient. For later usage we introduce the total number of MADS iterations $n_{I}$ as the sum of the number of steps in each pass.

\section{F. MADS-followup computing cost}

Contrary to grid-based searches, the computing cost of the MADS based algorithm is non-deterministic, due to the a-priori unknown number of explored parameter space points. To estimate the maximal computing cost of the refinement or the zoom stage using Eq. 19 , we need the maximal number of possibly evaluated templates

$$
\mathcal{N}_{\max }=\sum_{i=0}^{n_{I}} p_{\max }^{i},
$$

where $p_{\max }^{i}$ is the user-specified maximum of the number of computed templates at MADS iteration $i$. This maximal number is typically chosen large to avoid too early interruption of the MADS instance, e.g. when further improvement of the current solution is possible while the extremum is not yet found. However, if the extremum is found, a MADS iteration starting from this point terminates rapidly.

Note that the fundamental computing cost $c_{0}^{\mathrm{SFT}}$ in stochastic searches over the sky is typically larger than in a grid-based search, where a lot of templates with different spindown components can be computed at fixed sky position. This results in a larger value of about $c_{0}^{\mathrm{SFT}} \approx 3 \times 10^{-7} s$ instead of the number quoted in Sec. IIIC.

\section{G. False-alarm and detection probability}

After the final fully-coherent zoom stage we are left with a candidate falling in one of the three categories discussed earlier, namely: the candidate is consistent with the signal model $(S)$, with Gaussian noise $(G)$ or is of non-Gaussian origin but inconsistent with the signal model. An additional valuable piece of information is the false-alarm probability associated with the candidate. This is the probability of exceeding a threshold $2 \mathcal{F}$-value 
in the absence of a signal, where the relevant distribution is the central $\chi^{2}$ distribution with 4 degrees of freedom, denoted as $\chi_{4}^{2}(2 \mathcal{F})$. The single-template false-alarm probability is

$$
\begin{aligned}
p_{\mathrm{fA}}^{1} & =\int_{2 \mathcal{F}_{\mathrm{th}}}^{\infty} d(2 \mathcal{F}) \chi_{4}^{2}(2 \mathcal{F}) \\
& =\left(1+\mathcal{F}_{\mathrm{th}}\right) e^{-\mathcal{F}_{\mathrm{th}}},
\end{aligned}
$$

and for $\mathcal{N}$ independent templates this results in

$$
p_{\mathrm{fA}}=1-\left(1-p_{\mathrm{fA}}^{1}\right)^{\mathcal{N}},
$$

where for $\mathcal{N} p_{\mathrm{fA}}^{1} \ll 1$, Taylor expansion yields $p_{\mathrm{fA}} \approx$ $\mathcal{N} p_{\text {fA }}^{1}$. For example, a threshold of $\widetilde{2 \mathcal{F}}_{\text {th }}^{(G)}=70$ for a search with $\mathcal{N}=1 \times 10^{5}$ templates corresponds to a falsealarm probability of $p_{\mathrm{fA}} \lesssim 2 \times 10^{-9}$, where the upper bound corresponds to $\mathcal{N}$ completely independent templates.

The overall detection probability of the follow-up method depends on the signal SNR. Higher SNR in the refinement stage yields better localization of the signal, i.e. a smaller Fisher ellipse and thus also a higher probability of signal recovery (35). In addition, the MADSalgorithm parameters also affect the detection efficiency, e.g. an increased number of MADS iterations increases the detection probability, especially for signals with lower SNR. Because of this, the detection probability will have to be estimated empirically in a Monte Carlo study, see Figs. $4 \mathrm{c}$ and $5 \mathrm{c}$.

\section{MONTE CARLO STUDIES}

To demonstrate the capability of the systematic followup procedure proposed in Section III we perform two different types of Monte Carlo (MC) studies.

In the first case we simulate a so-called directed search for a fixed sky position, where we follow up candidates in a 2 -dimensional spindown space, i.e. $\{f, \dot{f}\}$. In the second case we simulate an all-sky search over the 4dimensional parameter space $\{\alpha, \delta, f, \dot{f}\}$.

All MADS searches are implemented using the MADS reference library NOMAD 29 and the LAL library from the LALSUITE [25] is used for the $\mathcal{F}$-statistic computation [30]. The Gaussian data and signal injections are produced using the LALAPPS programs from LALSUITE. In particular with lalapps_Makefakedata_v4 we create data sets of total duration $T=200 \mathrm{~d}$, with $N=200$ segments of duration $\Delta T=1 \mathrm{~d}$, using SFTs of length $T_{\mathrm{SFT}}=1800 \mathrm{~s}$, for the two LIGO detectors $\mathrm{H} 1$ and L1. The noise level per detector is generated as Gaussian white noise with a power-spectral density $S_{n}$ of $\sqrt{S_{n}}=2 \times 10^{-23} \mathrm{~Hz}^{-1 / 2}$.

Independently of the type of the search, the initial candidates to follow-up are prepared as follows. Rather than performing a semi-coherent grid based search using the Hough- 5] or GCT-method [6], we generate candidates by drawing a random point in the vicinity of the injection and consider it a candidate if the semi-coherent metric mismatch $\mu^{*}$ is within the range

$$
\mu^{*} \in[0,1],
$$

see Figs. 4a and 5a. This procedure for candidate preparation allows us to separate the study of the followup algorithm from the problem of how to setup a semicoherent search, which is a difficult question on its own.

Note that even if the original grid-based semi-coherent search does not produce candidates that conform with Eq. (45), we can always increase the density of the grid until (45) applies. This would amount to a (cheap) preprocessing stage inserted before the present follow-up procedure .

\section{A. Follow-up of candidates from a directed search}

For the directed type of searches we fix the sky position to the coordinates of the Galactic Center. This choice is arbitrary and we could use any other point without qualitatively changing the results. We create 5000 data sets. Note that each data set has different Gaussian noise realization in which a CW signal from an isolated source is injected. In the process of injection, the original noise data set is also used to examine the behavior of the follow-up method in the absence of a signal.

The pulsar injection parameters $\lambda_{s}$ are drawn uniformly in the range $f \in(50,51) \mathrm{Hz}, \cos \iota \in(-1,1)$, $\psi \in(-\pi / 4, \pi / 4)$ and $\phi_{0} \in(0,2 \pi)$. The signal amplitude $h_{0}$ is chosen such that the expected average-SNR ${ }^{2}$ of Eq. (7) for a perfect match is distributed uniformly in the range $\rho^{2}{ }_{s} \in(0,2)$. The spindown $\dot{f}$ is chosen uniformly in the range $\dot{f} \in\left(-\frac{f_{\min }}{\tau_{\min }}, \frac{f_{\min }}{\tau_{\min }}\right)$ with minimal spindown age $\tau_{\min }=300 \mathrm{yr}$ at $f_{\min } \stackrel{\tau_{\min }}{=} 50 \mathrm{~Hz}$. The MADS-algorithm parameters used in the MC are summarized in Table $\mathrm{I}$, which have been found empirically to achieve good results. For this type of follow-up we find that the 1st

\begin{tabular}{c|c|c|c|c|c} 
stage & $w^{-}$ & $w_{\min }^{+}$ & $w_{\max }^{+}$ & $u_{b}$ & $p_{\max }$ \\
\hline$R$ & -1 & 1 & 1 & 2 & 20000 \\
\hline$Z$ & -1 & 1 & 50 & 1.1 & 20000
\end{tabular}

TABLE I: Algorithm parameters for follow-up of candidates from directed searches.

pass of the search algorithm in the refinement stage and only two repetitions of the 2 nd pass in the zoom stage is sufficient. We restrict the size of the search box for the refinement stage $\Delta \lambda_{R}$ by taking 1 frequency and 2 first spindown metric extents. In the zoom stage we constrain the parameter space to a Fisher ellipse Eq. 25) with $n_{\mathrm{B}}=24$.

We first apply the follow-up chain to the pure Gaussian-noise data without injected signals. The corresponding $\widetilde{2 \mathcal{F}}_{Z}$ distribution of the resulting fully-coherent zoom stage is plotted in Fig. $4 \mathrm{~b}$. The maximal value 


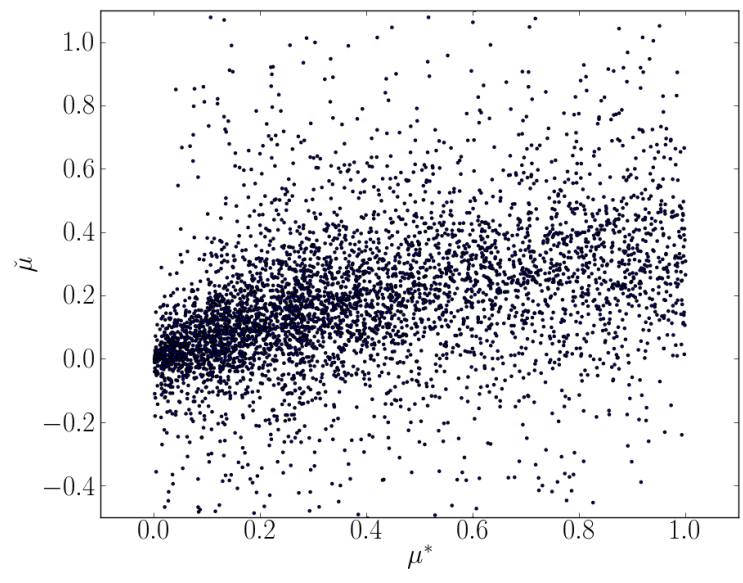

(a) SNR loss of the initial candidates $\check{\mu}$ versus semi-coherent metric mismatch $\mu^{*}$ to the closest template.

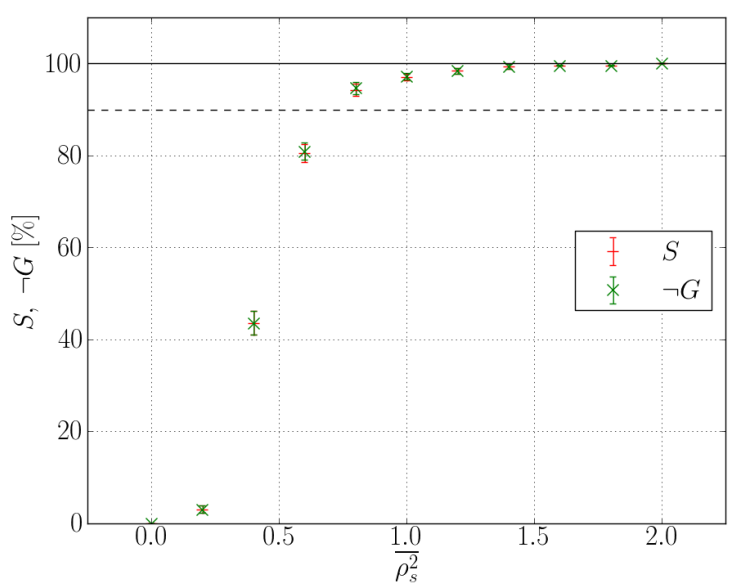

(c) Percentage of the 5000 injected signals classified as recovered $(-S)$ and of non-Gaussian origin $(\times \neg G)$ as

function of the non-centrality parameter $\overline{\rho_{s}^{2}}$, Eq. 77. The error

bars are computed by using a Jackknife estimator.

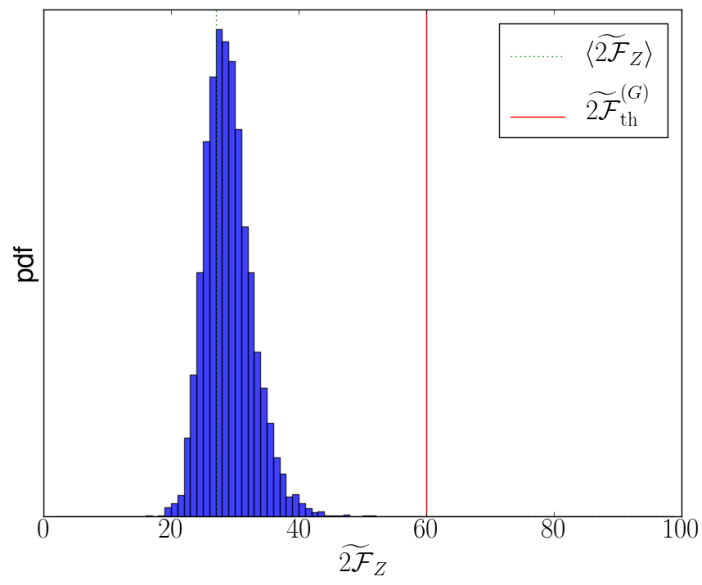

(b) $\widetilde{2 \mathcal{F}}_{Z}$ distribution after the fully-coherent 2-D $\{f, \dot{f}\}$ zoom stage of 5000 directed searches in pure Gaussian noise without injected signal. The maximal $2 \mathcal{F}$-value found is

$\widetilde{2 \mathcal{F}}_{Z}^{\text {max }}=51.61$. The mean value $\left\langle\widetilde{2 \mathcal{F}}_{Z}\right\rangle=29.00$ is plotted with a dotted line.
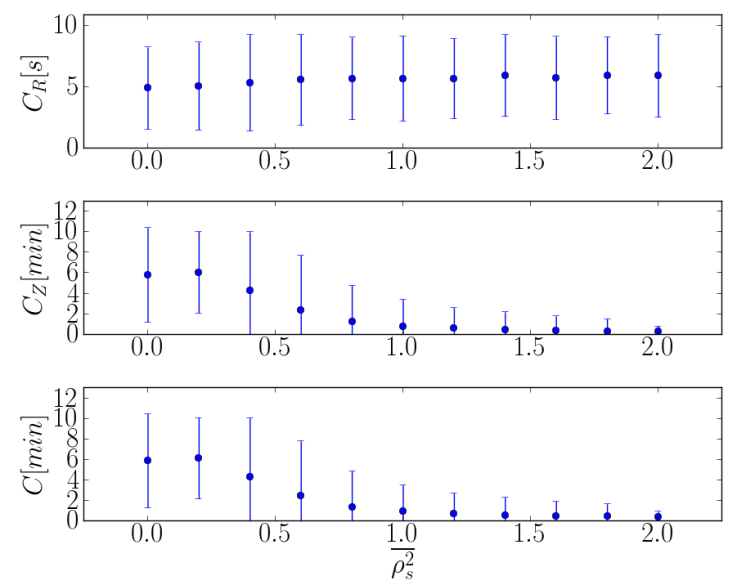

(d) Upper plot: computing cost of the semi-coherent refinement stage. Middle plot: computing cost of the fully-coherent zoom stage. Lower plot: total computing cost.

FIG. 4: Monte Carlo study of 2-stage follow-up of candidates from a directed $\{f, \dot{f}\}$ semi-coherent search pointed toward the Galactic Center with $N=200$ segments of duration $\Delta T=1 \mathrm{~d}$.

found is $\widetilde{2 \mathcal{F}}_{Z}^{\max }=51.61$. We therefore use a threshold for the classification of non-Gaussian candidates $(\neg G)$ of $\widetilde{2 \mathcal{F}}_{\text {th }}^{(G)}=60$, which is safely above this level.

We next apply the follow-up chain to the Gaussiannoise data with injected signal. In Fig. $4 \mathrm{c}$ we plot the percentage of injected signals that are classified as recovered signals $(S)$ and non-Gaussian origin $(\neg G)$, as a function of the injected signal strength $\overline{\rho^{2}}{ }_{s}$. From this plot we can read out the detection probability, namely we reach $90 \%$ of signal recovery for candidates with $\overline{\rho_{s}^{2}} \approx 0.7$.

The computing cost as function of $\overline{\rho_{s}^{2}}$ is plotted in Fig. $4 \mathrm{~d}$. We notice that the cost of the refinement stage is negligible and in the zoom stage the averaged computing time decreases with higher signal strength.

\section{B. Follow-up of candidates from an all-sky search}

The data and signal preparation for the following allsky Monte Carlo study is the same as in the directed search case, however the sky position is drawn isotropically over the whole sky. We create 7500 data sets with uniformly distributed injected average-SNR ${ }^{2}$ in the range $\overline{\rho_{s}^{2}} \in(0,3)$. The algorithm parameters used in the refine- 




(a) SNR loss of the initial candidates $\check{\mu}$ versus semi-coherent metric mismatch $\mu^{*}$.

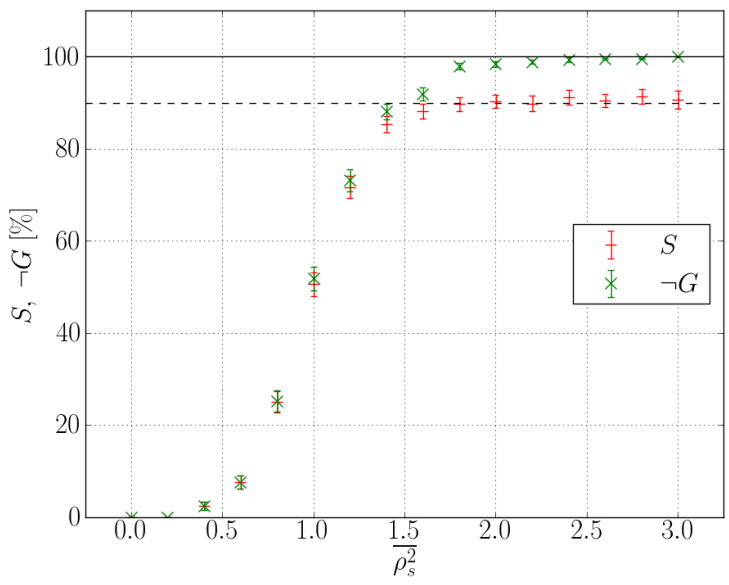

(c) Percentage of the 7500 injected signals classified as recovered $(-S)$ and of non-Gaussian origin $(\times \neg G)$ as function of the signal strength $\overline{\rho_{s}^{2}}$. The error bars are computed using a Jackknife estimator.



(b) $\widetilde{2 \mathcal{F}}_{Z}$ distribution after the fully-coherent 4 -D $\{\alpha, \delta, f, \dot{f}\}$ zoom stage of 7500 searches in pure Gaussian noise, without injected signal. The maximal $2 \mathcal{F}$-value found is $\widetilde{2 \mathcal{F}}_{Z}^{\max }=58.76$. The mean value is $\left\langle\widetilde{2 \mathcal{F}}_{Z}\right\rangle=37.50$ indicated with dots.
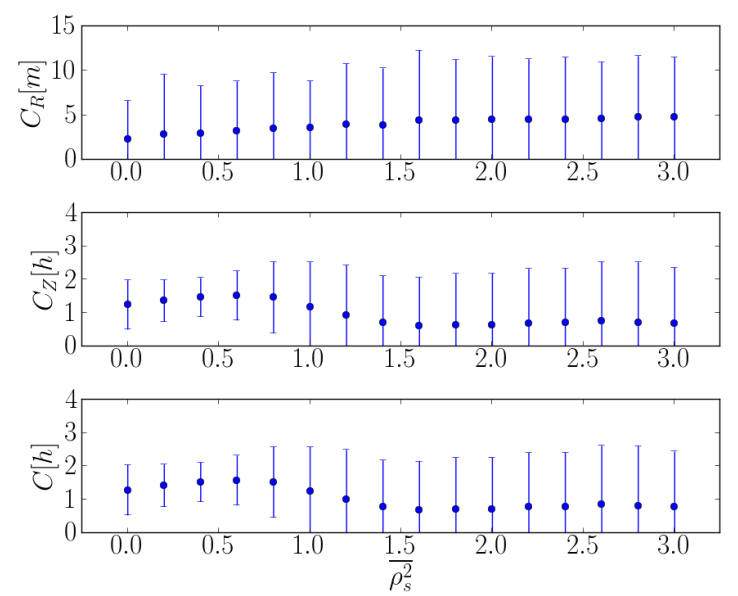

(d) Upper plot: computing cost of the semi-coherent refinement stage. Middle plot: computing cost of the fully-coherent zoom stage. Lower plot: total computing cost.

FIG. 5: Monte Carlo study of 2-stage follow-up of candidates from an all-sky $\{\alpha, \delta, f, \dot{f}\}$ semi-coherent search with $N=200$ segments of duration $\Delta T=1 \mathrm{~d}$.

ment and zoom stage are given in Table [I] which have been found empirically to yield good performance. We also find that here the zoom stage benefits from performing all 4 search passes shown in Fig. 3. The size of the search box for the refinement stage in the spindown subspace has been defined exactly as in the directed search example. The sky subspace is constrained by using an $m=1$ iso-mismatch ellipse. As in the previous example we use $n_{\mathrm{B}}=24$ in Eq. (25) to determine the size of the Fisher ellipse.

Similarly to the directed follow-up, we first test the pipeline using the Gaussian noise data without injections. The resulting distribution of final $\widetilde{2 \mathcal{F}}_{Z}$ values is plotted

\begin{tabular}{c|c|c|c|c|c} 
stage & $w^{-}$ & $w_{\min }^{+}$ & $w_{\max }^{+}$ & $u_{b}$ & $p$ \\
\hline$R$ & -1 & 1 & 5 & 2 & 20000 \\
\hline$Z$ & -1 & 1 & 50 & 1.2 & 20000
\end{tabular}

TABLE II: Follow-up algorithm parameters for full parameter space searches.

in Fig. $5 \mathrm{~b}$ The maximal value found is $\widetilde{2 \mathcal{F}}_{Z}^{\max }=58.76$, which is higher compared to the value found in the directed follow-up searches due to the increased number of evaluated templates. We therefore use a threshold for the classification of non-Gaussian candidates $(\neg G)$ of $\widetilde{2 \mathcal{F}}_{\text {th }}^{(G)}=70$, which is safely above this level. 
Next we search the data containing the injected signals. In Fig. $5 \mathrm{c}$ we plot the fraction of signals classified as recovered $(S)$ and the percentage of MC trials found to be of non-Gaussian origin $(\neg G)$, as a function of the injected signal strength $\overline{\rho^{2}}{ }_{s}$ In order to achieve $90 \%$ signal recovery $(S)$, we now need stronger signals, namely $\overline{\rho_{s}^{2}} \gtrsim 1.7$. However, for $\overline{\rho_{s}^{2}} \approx 1.5$ we can already achieve $90 \%$ "detection probability" in the sense of separating candidates from Gaussian noise $(\neg G)$. This indicates that the zoom step sometimes converges on a secondary maximum. Given that any non-Gaussian $(\neg G)$ candidates after zoom will receive further scrutiny, it would be straightforward to further explore the parameter space around such candidates to localize a potential primary maximum.

The computing cost as a function of $\overline{\rho_{s}^{2}}$ is plotted in Fig. $5 \mathrm{~d}$. We notice that the total computing cost is dominated by the zoom stage and the averaged computing time is rather independent of the signal strength.

\section{DISCUSSION}

We have studied a two-stage scheme for the fullycoherent follow-up of semi-coherent candidates. The first stage, called refinement, aims to find the maximumlikelihood estimator of the initial semi-coherent candidate. This allows us to better constrain the parameter space for the coherent zoom stage. The two-stage scheme is suitable for following-up candidates from all-sky or di- rected semi-coherent searches. The proposed grid-less optimization lowers the computing cost per candidate to acceptable levels. In Monte Carlo studies we tested the efficiency of the algorithm for directed and all-sky followup searches.

In this paper we restricted the all-sky follow-up optimization to 4 dimensions, namely sky, frequency and first spindown. Further work is required to extend the optimization to higher dimensions. A related attractive direction for further development is the extension and application of the search algorithm for follow-up of CW candidates in binary systems, which is a challenging higher-dimensional problem.

We also aim to extend the two-stage scheme presented here by including intermediate semi-coherent zoom stages. This should allow to further reduce the computing cost and increase detection efficiency.

\section{ACKNOWLEDGMENTS}

We are grateful to numerous colleagues for useful comments and discussions, in particular Karl Wette, Holger Pletsch, Paola Leaci, Vladimir Dergachev, Thomas Dent, Badri Krishnan, Maria Alessandra Papa and Bruce Allen. MS gratefully acknowledges the support of Bruce Allen and the IMPRS on Gravitational Wave Astronomy of the Max-Planck-Society. This paper has been assigned LIGO document number LIGO-P1200185-v2.
[1] P. Jaranowski, A. Krolak, and B. F. Schutz, Phys. Rev. D58, 063001 (1998), gr-qc/9804014.

[2] P. R. Brady, T. Creighton, C. Cutler, and B. F. Schutz, Phys. Rev. D 57, 2101 (1998).

[3] P. R. Brady and T. Creighton, Phys. Rev. D61, 082001 (2000), gr-qc/9812014.

[4] C. Cutler, I. Gholami, and B. Krishnan, Phys. Rev. D 72, 042004 (2005).

[5] B. Krishnan et al., Phys. Rev. D 70, 082001 (2004).

[6] H. J. Pletsch and B. Allen, Phys. Rev. Lett. 103, 181102 (2009).

[7] B. Abbott et al. (LIGO Scientific Collaboration), Phys. Rev. D 77, 022001 (2008).

[8] J. Aasi et al. (The LIGO Scientific Collaboration and the Virgo Collaboration) (2012), 1207.7176.

[9] Einstein@home, http://www.einsteinathome.org (2011).

[10] R. Prix and M. Shaltev, Phys. Rev. D 85, 084010 (2012), URL http://link.aps.org/doi/10.1103/PhysRevD. 85.084010.

[11] P. Jaranowski and A. Krolak, Phys.Rev. D61, 062001 (2000), gr-qc/9901013.

[12] A. Krolak, M. Tinto, and M. Vallisneri, Phys.Rev. D70, 022003 (2004), gr-qc/0401108.

[13] C. Cutler and B. F. Schutz, Phys. Rev. D 72, 063006 (2005).
[14] B. J. Owen, Phys. Rev. D 53, 6749 (1996).

[15] R. Balasubramanian, B. S. Sathyaprakash, and S. V. Dhurandhar, Phys. Rev. D 53, 3033 (1996).

[16] M. Vallisneri, Phys. Rev. D 77, 042001 (2008), URL http://link.aps.org/doi/10.1103/PhysRevD.77. 042001

[17] R. Prix, Phys. Rev. D 75, 023004 (2007), gr-qc/0606088.

[18] S. M. Kay, Fundamentals of Statistical Signal Processing, Volume I: Estimation Theory (v. 1) (Prentice Hall, 1993), 1st ed., ISBN 0133457117, URL http://www.worldcat. org/isbn/0133457117.

[19] D. Coe (2009), 0906.4123.

[20] P. R. Williams and B. F. Schutz (1999), arXiv:grqc/9912029

[21] R. Prix, Class. Quant. Grav. 24, S481 (2007).

[22] C. Messenger, R. Prix, and M. A. Papa, Phys. Rev. D 79, 104017 (2009).

[23] H. J. Pletsch, Phys. Rev. D 82, 042002 (2010), 1005.0395.

[24] B. Abbott, R. Abbott, R. Adhikari, P. Ajith, B. Allen, G. Allen, R. Amin, D. P. Anderson, S. B. Anderson, W. G. Anderson, et al. (LIGO Scientific Collaboration), Phys. Rev. D 79, 022001 (2009), URL http://link.aps . org/doi/10.1103/PhysRevD.79.022001.

[25] lalsuite, https://www. Isc-group.phys .uwm.edu/daswg/ projects/lalsuite.html (2011).

[26] C. Audet and J. E, SIAM Journal on optimization 17, 
2006 (2004).

[27] M. A. Abramson, C. Audet, J. E. Dennis, and S. L. Digabel, SIAM Journal on Optimization 20, 948 (2009), URL http://dblp.uni-trier.de/db/journals/ siamjo/siamjo20.html\#AbramsonADD09.

[28] A. R. Conn and S. L. Digabel, Optimization methods and software (2011).

[29] S. Le Digabel, ACM Trans. Math. Softw. 37, 44:1 (2011), ISSN 0098-3500, URL http://doi.acm.org/10.1145/ 1916461.1916468

[30] R. Prix, Tech. Rep. (2010), LIGO-T0900149-v3. 